Relations industrielles

Industrial Relations

\title{
Étude comparative sur la législation de conciliation et d'arbitrage
}

\section{Anonyme}

Volume 6, numéro 1, décembre 1950

URI : https://id.erudit.org/iderudit/1023246ar

DOI : https://doi.org/10.7202/1023246ar

Aller au sommaire du numéro

Éditeur(s)

Département des relations industrielles de l’Université Laval

ISSN

0034-379X (imprimé)

1703-8138 (numérique)

Découvrir la revue

Citer cet article

Anonyme (1950). Étude comparative sur la législation de conciliation et d'arbitrage. Relations industrielles / Industrial Relations, 6(1), 11-23.

https://doi.org/10.7202/1023246ar

Tous droits réservés @ Département des relations industrielles de l’Université Laval, 1950
Ce document est protégé par la loi sur le droit d'auteur. L'utilisation des services d'Érudit (y compris la reproduction) est assujettie à sa politique d'utilisation que vous pouvez consulter en ligne.

https://apropos.erudit.org/fr/usagers/politique-dutilisation/ 
nus dans les CTF en Tchécoslovaquie a été envoyée en URSS, et que d'autres attendent le même sort. Le centre de déportation de Tchécoslovaquie en URSS semble être le camp de Leopoldov, dans les Sudètes.

Nous avons exposé ici, en autant que nos renseignements nous le per- mettaient, les conditions qui prévalent dans les camps de travaux forcés de Tchécoslovaquie de même que les raisons officielles de l'établissement de ces camps. Nous nous proposons de donner dans un article subséquent les raisons réelles de l'établissement des camps de travaux forcés.

\section{Etude comparative sur la législation de conciliation et d'arbitrage}

\section{INTRODUCTION}

La présente étude ne constitue pas un exposé des principes qui sont à la base des divers systèmes de règlement des conflits du travail, mais simplement une analyse, une description des principaux types de systèmes de règlement, sans que soient portés des jugements de valeur sur les uns et les autres. Cependant quelques précisions sont nécessaires.

Par «conflits du travail» nous envisageons ici seulement les conflits collectifs et non les conflits individuels, bien que ceux-ci peuvent très souvent se transformer en conflits collectifs. D'ailleurs la distinction entre conflits individuels et conflits collectifs présente plutôt un caractère artificiel comme le font remarquer Rouast et Durand dans leur «Précis de législation industrielle . $^{1}$ Toutefois, s'il n'est pas facile d'établir les critères d'un conflit individuel, il est plus facile de reconnaître les caractéristiques d'un conflit collectif du travail.

(1) Rouast et Durand, Précis de législation industrielle (Droit du Travail), p. 276 et suivantes, Librairie Dalloz, 1948.

\section{Note de la rédaction:}

Sous ce titre nous publierons dans la rovue une série d'articles rédigés par un professeur du Département des relations industrielles de Laval qui à cause de certaines circonstances particulières désire garder l'anonymat.
A ce sujet, Rouast et Durand adoptent la position suivante: «a) Constituent des conflits collectifs, ceux qui mettent en jeu une question de principe, dont la solution intéresse la condition juridique des différents membres d'un groupement. Il en est d'abord ainsi quand le conflit est relatif à la création ou à la revision d'une règle du droit du travail. (Etablissement d'un règlement arbitral des conditions du travail, revision de la convention collective, d'une sentence arbitrale pour cause d'imprévision). De même, présente un caractère collectif le conflit relatif à l'interprétation des règles du droit, qu'il s'agisse des lois, d'usages, de conventions collectives ou de sentences arbitrales. b) Constituent en second lieu des conflits collectifs ceux qui mettent en jeu un intérêt commun à toute ou partie de la collectivité, qui intéressent par exemple la liberté d'opinion, la liberté syndicale, les droits des délégués du personnel, le recours à la grève, même si la solution du différend doit modifier la situation juridique d'un seul membre de l'entreprise. Une mesure individuelle a donc pu donner naissance d un conflit collectif: il suffit de supposer un congédiement, motivé par l'adhésion du salarié à un syndicat. En portant atteinte au droit syndical, l'acte lèse une prérogative de la 
collectivité ouvrière. La compétence de la juridiction arbitrale ne pouvait d'ailleurs résulter d'une simple allégation, mais de la preuve d'une atatteinte portée à l'intérêt collectif. La compétence et le fond du litige étaient intimement liés. La notion du conflit collectif est donc très large, limitant celle du conflit individuel ». ${ }^{2}$ Les systèmes de règlement qui sont analysés dans cette étude ressortissent aux conflits qui présenteront les caractéristiques soulignées par Rouast et Durand, qu'ils s'agissent des conflits de droit ou des conflits d'intérêts (économiques).

Beaucoup plus importante est la distinction entre conflits de droit et conflits d'intérêts au point de vue de l'examen des systèmes de règlement des conflits collectifs du travail, car très souvent cette distinction conduit a l'établissement de tribunaux du travail chargés de régler les conflits de droit, notamment dans les pays scandinaves, tandis que les conflits d'intérêts relèvent de la procédure de conciliation et d'arbitrage. En outre, même si cette distinction ne conduit pas à l'établissement de tribunaux du travail, elle peut conduire à l'interdiction de la suspension du travail à propos d'un conflit de droit, notamment en matière d'interprétation et d'application des conventions collectives, conflit qui dans ce cas doit être réglé définitivement par une procédure inscrite dans la convention collective, comme le prévoit par exemple le code fédéral du travail canadien.

Le conflit de droit « porte sur l'interprétation ou l'application d'un droit né et actuel, peu importe que celle-ci ait sa source dans une prescription formelle de la loi ou dans une disposition d'un contrat individuel ou collectif. Son interprétation relève normalement du juge, en l'espèce du juge du travail $»^{3}$ Les con-

(2) Idem.

(3) Les tribunaux du travail, p. 20, Etudes et Documents, Série A (Vie sociale). No 40, Bureau international du Travail, Genève, 1948. flits de droit dont il est fait mention dans cette étude concernent principalement ceux qui résultent de l'interprétation et de l'application du droit du travail et des conventions collectives. La question de savoir si les conflits de droit doivent ressortir à une juridiction spéciale (tribunaux du travail) ou à la juridiction ordinaire n'entre pas dans le cadre de cette étude. Nous nous limiterons à décrire les tribunaux du travail qui existent dans les pays dont les systêmes de règlement des conflits du travail sont analysés dans cette étude. ${ }^{*}$

Le conflit d'intérêts «porte non pas sur l'interprétation d'un droit acquis fondé sur la loi ou le contrat, mais sur une simple revendication tendant à modifier un droit existant ou à créer un droit nouveau. Ce genre de conflit relève normalement

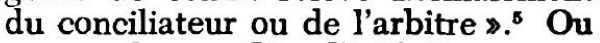
encore, les conflits d'intérêts - que plusieurs appellent conflits économiques - comprennent les conflits «provoqués par des divergences fondamentales en matière de salaires et de conditions de travail, c'est-à-dire par des questions qui sont normalement réglées par des conventions collectives $\gg .^{6}$

Les systèmes de règlement des conflits du travail analysés dans cette étude appellent donc un examen des mécanismes de conciliation et d'arbitrage et des tribunaux du travail qui existent dans les pays que nous avons choisis. Précisons dès maintenant que la règle qui a présidé à ce choix visait simplement à mettre en lumière les divers systèmes en pratique et non à présenter une analyse complète et définitive de tous les systèmes en vigueur dans tous les pays. Les pays choisis l'ont été à titre d'exemple et non parce que

(4) Idem.

(5) Idem.

(6) Relations industrielles, Rapport VIII -1-, p. 93, Conférence internationale du Travail, Trente et unieme session, San Francisco, 1948. 
les systèmes qu'ils appliquent sont les meilleurs. D'ailleurs en matière de règlement des conflits du travail il existe des systèmes et non une doctrine unique.

En effet, les systèmes de règlement des conflits du travail varient fondamentalement selon la structure de l'économie des divers pays. «Lorsqu'il s'agit d'un pays ayant une économie planifiée où les relations entre la direction de l'industrie et les travailleurs doivent être stabilisées et réglementées dans le cadre de la structure industrielle, laquelle est subordonnée à la réalisation du plan économique national, le problème du règlement des conflits du travail qui pourraient surgir à l'occasion de la conclusion d'une convention collective a des aspects différents de ceux qu'il présente dans une économie libre $\gg .^{7}$ Il est évident que l'économie entièrement planifiée exige des modes de règlement qui ont un caractère obligatoire, tant au point de vue des procédures possibles que des décisions qui sont prises. Conflits d'intérêts aussi bien que conflits de droit ressortissent à des instances dont les décisions sont obligatoires, bien que dans certains pays à économie planifiée le droit de grève n'ait pas été restreint par la loi.

Au contraire, dans les pays de capitalisme libéral ou dans les pays qui, tout en possédant un large secteur d'économie planifiée, pratiquent la libre concurrence, la liberté des prix et des salaires, les systèmes de règlement des conflits du travail ne présentent pas le même caractère de coercition. Dans certains de ces pays, les conflits de droit ressortissent tantôt à la juridiction ordinaire, tantôt à une juridiction spéciale (tribunaux du travail). Bien qu'on ne puisse affirmer que la tendance soit vers le règlement définitif des conflits de droit (par des tribunaux du travail ou par d'autres organismes ou procédures) sans que l'interruption du tra-

(7) Idem, p. 125. vail soit générale, il semble cependant que c'est vers cette voie que s'acheminera la pratique des relations industrielles.

Quant aux conflits d'intérêts (ou conflits économiques) une tendance bien précise se dessine: obligation de recourir à des procédures de conciliation et à des procédures d'arbitrage. Toutefois nous rencontrons ici plusieurs variétés de systèmes. En effet, dans certains pays, seule la procédure de conciliation avant l'action directe, tant de la part des syndicats que des employeurs, est obligatoire, soit en vertu des conventions, soit en vertu de la loi. Il va de soi qu'en pareil cas les conclusions de la procédure de conciliation n'ont pas un caractère obligatoire Dans d'autres pays, un deuxième stade est prévu: la procédure d'arbitrage. Dans certains cas, le recours à cette procédure est obligatoire mais les conclusions n'obligent pas les parties. Dans d'autres cas, la législation n'oblige pas les parties à recourir à cette procédure, mais les y incite seulement, étant donné que les sentences doivent alors être respectées.

Quelle différence existe-t-il entre la procédure de conciliation et la procédure d'arbitrage ? La conciliation tend « à recréer la volonté même des parties», tandis que l'arbitrage tend «à suppléer à la volonté manquante $\gg .^{8}$ La première méthode aboutit «à l'accord des parties», tandis que l'autre aboutit à la «décision d'un tiers qui se substitue à l'accord $\gg .^{9}$

$\mathrm{La}$ procédure de conciliation, qu'elle soit conventionnelle ou obligatoire, c'est-à-dire établie par les parties ou prévue par la législation, constitue donc le premier stade. L'intervention éventuelle du conciliateur

(8) Méthodes de collaboration entre les pouvoirs publics, les organisations professionnelles ouvrières et les organisations professionnelles patronales, p. 160, Vingtsixième session, Conférence internationale du Travail, Genève, 1940.

(9) Idem. 
a pour but d'aider les parties à aboutir par elles-mêmes à un accord et non à leur imposer une solution. En d'autres termes, la procédure de conciliation ne donne pas lieu à une sentenco comme l'arbitrage qui au contraire exprime un jugement, une règle qui devrait être appliquée. Tous les pays accordent une grande importance a la procédure de conciliation au point qu'une sorte de priorité est accordée, même dans les pays où existe la conciliation obligatoire, aux mécanismes de conciliation qui peuvent avoir été prévus par les parties elles-mêmes.

La procédure d'arbitrage a un caractère beaucoup plus ferme, qu'il s'agisse de l'arbitrage conventionnel ou de l'arbitrage obligatoire. Alors qu'en règle générale la procédure de conciliation est plutôt vague, la procédure d'arbitrage est très précise: nomination des arbitres ou plus exactement des représentants du syndicat et de l'employeur, désignation du président de la commission ou du tribunal d'arbitrage, qui en fait est la véritable arbitre ou le surarbitre, comparution des parties, dépôt des documents, assignation des témoins, etc.

En analysant les divers systèmes de règlement des conflits du travail établis par la législation, on constate le souci constant de l'autorité publique de n'intervenir que pour « rétablir la volonté déficiente des parties». A tous les stades de la procédure, soit de la conciliation, soit de l'arbitrage, il est toujours loisible aux parties d'en venir à un accord par des voies qu'elles choisissent elles-mêmes.

Le grand mérite des procédures de conciliation et d'arbitrage est de réglementer la grève et le lock-out. Avant de recourir à l'action directe, les parties sont obligées d'explorer toutes les possibilités de compromis et d'entente. Sans porter atteinte au droit de grève ou de lock-out, elles en conditionnent l'exercice.

Dans les pays où la liberté d'entreprise, la liberté des prix et la libre fixation des salaires sont la règle gé- nérale, la tendance n'est pas encore au règlement obligatoire des conflits d'intérêts. Pour les conflits de droit, la situation est quelque peu différente, car la procédure de règlement définitif sans interruption du travail gagne du terrain. $\mathbf{Y}$ a-t-il lieu de regretter le fait que la plupart des pays aient renoncé à l'arbitrage obligatoire (avec sentence obligatoire) des conflits d'intérêts? Il semble bien que recourir au caractère obligatoire des sentences arbitrales en matière de conflits d'intérêts, sauf pour certains secteurs de l'économie comme certains services publics, signifierait accepter non seulement l'interdiction de la grève et du lock-out, mais également une économie entièrement planifiée.

La valeur et l'efficacité des sentences arbitrales seraient-elles réduites du fait qu'elles ne sont pas obligatoires pour les parties? L'affirmer serait oublier le très fort caractère moral des sentences arbitrales par suite de leur caractère officiel et de la publicité qui accompagne leur publication. L'examen des rapports des services de conciliation et d'arbitrage des ministères du travail des divers pays démontrerait, nous en sommes convaincu, que dans la grande majorité des cas les sentences des commissions ou des tribunaux d'arbitrage sont en fait acceptées et appliquées par les parties, bien que la loi ne les oblige pas.

En l'état actuel des principes qui sont à la base de l'économie de la plupart des pays et qui gouvernent les relations industrielles, il semble que les divers systèmes de règlement des conflits du travail évolueront dans le cadre qui est le leur présentement.

Avant de passer à l'analyse de quelques-uns des systèmes de règlement des conflits du travail qui sont réellement représentatifs de la pratique suivie par divers pays, nous tenons à préciser qu'il n'a été tenu compte que des grandes lignes de ces systèmes, vu que le but de cette étu- 
de n'est que de donner une vue très générale des systèmes en vigueur. Dans la mesure du possible, nous nous sommes tenu le plus près possible du texte de la loi afin d'éviter toute confusion. Enfin, nous avons tenu compte de la législation de base et non des lois qui peuvent s'appliquer à certaines industries particulières ou aux services publics.

\section{CANADA}

\section{A-LEGISLATION FEDERALE}

Il existe deux lois en matière de règlement des conflits du travail: la loi sur les relations industrielles et sur les enquêtes visant les différends du travail $^{1}$ et la loi concernant la conciliation et le travail ${ }^{2}$ qui s'applique aux chemins de fer.

\section{I-Loi sur les relations industrielles et sur les enquêtes visant les différends du travail}

Cette loi adoptée par la Chambre des Communes le 17 juin 1948 et entrée en vigueur le ler septembre de la même année «est en grande partie une révision des règlements des relations ouvrières en temps de guerre, avec les modifications et additions qui ont paru opportunes à la lumière de l'expérience acquise dans l'application des règlements et des représentations reçues des autorités provinciales et des organisations patronales et ouvrières $\gg .^{3}$

\section{a) Champ d'application}

La loi s'applique aux travailleurs employés aux ouvrages, entreprises ou affaires qui relèvent de l'autorité législative du Parlement du Canada

(1) Loi tendant à l'examen, à la conciliation et au règlement des différends du travail, 11-12 Geo. VI, ch. 54, 1948.

(2) Loi concernant la conciliation et le travail, Chapitre 110, S.R. du Canada, 1927.

(3) Gazette du Travail, Vol. XLVII, no 7, juillet, 1947, p. 962, Ministère du Travail, Ottawa. (art. 53). Toutefois, lorsque la législation adoptée par la législature d'une province et la Partie I de la loi sont sensiblement uniformes, le ministre du Travail peut, au nom du gouvernement du Canada et avec l'approbation du gouverneur en conseil, conclure un accord avec le gouvernement de la province en vue de pourvoir à l'application de la législation provinciale par les fonctionnaires et employés du Canada (art. 62).

\section{b) Structure de la loi}

La loi comporte des dispositions concernant le droit d'affiliation à un syndicat, les pratiques déloyales en matière de travail, les négociations collectives et l'accréditation d'agents négociateurs par le Conseil des relations ouvrières, les conventions collectives, les grèves et les lock-outs, la conciliation et l'établissement de commissions d'enquêtes chargées de connaître des matières qui font l'objet de différends du travail. Nous nous limiterons à l'examen des dispositions de la loi qui concernent le règlement des conflits du travail.

\section{c) Négociation des conventions collectives}

Lorsqu'un avis d'entamer des négociations collectives a été donné aux termes de la loi et que i) des négociations collectives n'ont pas été commencées dans le délai prescrit par la loi ou que ii) des négociations collectives ont été commencées, l'une des parties peut intervenir auprès du Ministre du Travail aux fins suivantes:

\section{i) Nomination dun ou plusieurs conciliateurs}

L'une des parties peut demander au Ministre de charger un conciliateur de conférer avec les parties aux négociations pour les aider à conclure une convention collective. Après examen des difficultés rencontrées, le 
Ministre peut, s'il estime qu'il convient de le faire, charger un ou plusieurs conciliateurs d'assister les parties.

\section{ii) Nomination d'une commission de conciliation}

Lorsqu'un conciliateur ne réussit pas à amener les parties à une entente ou dans tout autre cas où le Ministre estime qu'une commission de conciliation devrait être nommée pour tenter d'aboutir à un règlement, le Ministre peut nommer à cette fin une commission de conciliation.

iii) Délais nécessaires avant de recourir à la grève ou au lock-out

L'article 21 de la loi précise les délais qui doivent être observés avant que les parties puissent recourir à l'action directe: «Lorsqu'un syndicat ouvrier, au nom d'une unité d'employés, a droit, moyennant un avis prévu par la présente loi, d'exiger que leur employeur entame des négociations collectives en vue de la conclusion, du renouvellement ou de la révision d'une convention collective, le syndicat ouvrier ne doit prendre aucun vote de grève, ni autoriser la prise d'un vote de grève d'employés dans l'unité, ou y participer, ni déclarer ou autoriser une grève des employés de l'unité, et nul employé de I'unité ne doit faire la grève, ni l'employeur déclarer ou causer un lockout des employés de l'unité, avant que a) l'agent négociateur et l'employeur ou leurs représentants autorisés à cet égard, aient négocié collectivement et manqué à conclure une convention collective; et b) qu'une commission de conciliation ait été nommée pour tenter d'amener une entente entre eux et que sept jours se soient écoulés depuis la date où le Ministre a reçu le rapport de la commission de conciliation, ou c) que l'une ou l'autre des parties ait demandé au Ministre, par écrit, de nommer une commission de conciliation pour tenter d'amener une en- tente entre elles et que quinze jours se soient écoulés après la date où le ministre a reçu ladite demande, et sauf si (i) aucun avis prévu au paragraphe deux de l'article vingt-huit* de la présente loi n'a été donné par le Ministre; ou (ii) le Ministre a avisé la partie qui a fait cette demande de sa décision de ne pas nommer une commission de conciliation ».

\section{d) Revision d'une stipulation de la convention susceptible de revision pendant la durée de la convention aux termes mêmes de celle-ci}

Les dispositions prévues précédemment, c'est-à-dire à c, (iii) s'appliquent également en cette circonstance.

e) Renouvellement ou revision de la convention ou conclusion d'une nouvelle convention collective

L'une ou l'autre partie à une convention collective peut, dans la période de deux mois précédant immédiatement la date où expire la durée de la convention ou précédant celle où il $y$ est mis fin, requérir, au moyen d'un avis, l'autre partie à la convention d'entamer des négociations collectives en vue du renouvellement ou de la revision de la convention ou de la conclusion d'une nouvelle convention collective.

«S'il n'a été conclu aucun renouvellement ou revision de la convention ni aucune nouvelle convention avant qu'expire la durée de la convention ou qu'il y soit mis fin, l'employeur ne doit pas, sans consentement donné par les employés visés ou en leur nom, réduire les taux de salaire, ni modifier aucune autre condition d'emploi en vigueur immédia-

(*) Art. 28 (2) «Lorsque le Ministre a décidé de nommer une commission de conciliation, il doit immédiatement, par écrit, requérir chacune des parties de désigner, dans les sept jours de la réception de l'avis par la partie, une personne pour membre de la commission de conciliation, et le $\mathrm{Mi}$ nistre sur réception de la désignation dans les sept jours nommera cette personne membre de la commission de conciliation. $\gg$ 
tement avant que ladite convention soit expirée ou qu'il $y$ soit mis fin selon les stipulations y contenues, tant qu'un renouvellement ou une revision de la convention ou une nouvelle convention collective n'aura pas été conclue, tant qu'une commission de conciliation, nommée pour tenter d'effectuer une entente, n'aura pas fait rapport au Ministre et que sept jours ne se seront pas écoulés après la réception du rapport par le Ministre, selon celui des deux faits qui est antérieur à l'autre, ou tant que le Ministre n'aura pas informé l'employeur qu'il a décidé de ne pas nommer une commission de conciliation 》 [art. 15 (b)].

De même, pendant ce temps, tout vote de grève, toute grève ou tout lock-out est interdit.

\section{f) Règlement des conflits de droit sans suspension du travail}

La loi contient des dispusitions prévoyant l'interdiction de toute suspension du travail à propos de l'interprétation ou de la violation d'une convention collective. Tel est le sens de l'article 19: «1) Toute convention collective conclue après l'entrée en vigueur de la présente loi doit contenir une disposition pour le règlement définitif, sans suspension de travail, par arbitrage ou autrement, de tous différends entre les parties à la convention ou entre les personnes liées par cette dernière ou au nom de qui la convention a été conclue, concernant le sens ou la violation de la convention. 2) Lorsqu'une convention collective, conclue avant ou après l'entrée en vigueur de la présente loi, ne contient pas la disposition requise par le présent article, le Conseil doit, par la demande de l'une ou l'autre partie, à la convention, au moyen d'une ordonnance, prescrire une disposition à cette fin. Une disposition ainsi prescrite est censée être une condition de la convention collective et lier les parties à la convention et à toutes les personnes liées par cette dernière ainsi que les personues au nom de qui la convention a été conclue. 3) Toute partie à la convention, toute personne liée par cette dernière, de même que toute personne au nom de qui la convention a été conclue, doit observer la disposition visant au règlement définitif, contenue dans la convention, et $y$ donner effet $\gg$.

\section{g) Interdiction de la grève et du lock-out}

Sauf en ce qui concerne la revision d'une stipulation d'une convention susceptible de revision pendant la durée de la convention aux termes mêmes de celle-ci, aucun employeur lié par la convention ne peut déclarer ou causer un lock-out, de même qu'aucun employé lié par une convention collective ou au nom de qui une convention collective a été conclue, ne peut faire la grève, et aucun agent négociateur qui est partie à la convention ne doit déclarer ou autoriser une grève de tout semblable employé, pendant la durée de la convention collective.

De même, nul employé dans une unité ne peut faire la grève tant qu'un agent négociateur n'a pas acquis le droit, au nom de l'unité d'employés, d'enjoindre à leur patron, par un avis prévu en la présente loi, d'entamer des négociations collectives en vue de la conclusion, du renouvellement ou de la revision d'une convention, et tant que les dispositions de la loi à cet égard n'ont pas été observées. Nul patron ne peut déclarer ni causer de lock-out d'employés pendant qu'une demande d'accréditation d'un agent négociateur, pour agir au nom de ces employés, est en instance devant le Conseil.

\section{h) Sentence exécutoire}

La loi stipule qu'après la nomination d'une commission de conciliation qu'il est possible, à toute époque, avant ou après la présentation du rapport de la commission, de décider que la recommandation de la com- 
mission liera les parties et qu'elles devront y donner suite, si les parties en décident ainsi.

\section{i) Procédure en matière de conciliation}

\section{1-Conciliateurs}

Lorsqu'un conciliateur est chargé de conférer avec les parties engagées dans des négociations collectives, il doit, dans les quatorze jours après qu'il a reçu ses instructions, ou dans un délai plus long qui peut être autorisé par le ministre, faire rapport a) indiquant les matières sur lesquelles les parties sont d'accord; b) exposant les matières, s'il en est, sur lesquelles les parties ne peuvent s'entendre; et c) concernant l'opportunité de nommer une commission de conciliation en vue d'aboutir à une entente.

\section{2-Commissions de conciliation}

Chaque commission de conciliation se compose de trois membres. Sur réception d'un avis du Ministre, chaque partie doit désigner, dans les sept jours, une personne qui devient membre de la commission. Sur réception de cette désignation le ministre, dans un délai de sept jours, nomme cette personne membre de la commission de concilation. Si une des parties néglige de désigner une personne, le ministre procède à cette désignation à sa place. Les deux membres désignés et nommés ont un délai de cinq jours pour désigner une troisième personne qui agira comme président de la commission de conciliation. Si la troisième personne n'est pas désignée dans le délai prescrit, le ministre procède à cette désignation. La loi précise les raisons qui peuvent rendre inéligible une personne comme membre d'une commission de conciliation.

\section{3-Procédure}

Sauf disposition contraire à la loi, une commission de conciliation établit sa propre procédure, mais doit fournir à toutes les parties l'occasion voulue de soumettre une preuve et de présenter des observations.

La décision d'une majorité des membres présents à une séance constitue la décision de la commission et, en cas de partage égal, la voix du président est prépondérante. Le quorum est constitué par la présence du président et d'un autre membre de la commission.

Le rapport de la majorité des membres constitue le rapport de la commission de conciliation.

La commission possède le pouvoir de citer des témoins et d'obtenir les documents et choses que la commission estime indispensables à sa tâche, mais les renseignements ainsi obtenus de ces documents ne peuvent être rendus publics, sauf dans la mesure où la commission de conciliation le juge opportun. Les membres de la commission et les personnes autorisées par celle-ci peuvent visiter les lieux.

\section{4-Rapport}

Chaque commission de conciliation doit faire connaître ses conclusions et recommandations au Ministre dans les quatorze jours qui suivent la nomination de son président, ou dans tel délai prorogé qui peut être convenu entre les parties ou que le Ministre peut à l'occasion accorder. Nul rapport d'une commission de conciliation, nul témoignage ou procédure devant une commission de conciliation n'est admissible en preuve dans aucune cour du Canada, saut s'il s'agit de poursuites pour parjure.

\section{j) Application de la loi}

La loi détermine les pénalités qui peuvent être imposées à la suite de violation des dispositions de la loi. Cependant, il ne peut être intenté de poursuites pour une infraction visée par la loi qu'avec le consentement par écrit du Ministre. 


\section{k) Enquêtes en matière industrielle}

La loi prévoit encore que le ministre peut, sur demande ou de son propre chef, procéder ou faire procéder aux enquêtes qu'il croit utiles sur des questions industrielles, et il peut accomplir les choses qui paraissent propres à maintenir ou à garantir la paix industrielle et à faciliter des conditions favorables au règlement des différends.

Une commission d'enquête industrielle se compose d'un ou plusieurs membres nommés par le ministre. Elle a des pouvoirs identiques à ceux des commissions de conciliation en matière de citation de témoins, $d$ 'accès dans les lieux de travail et d'inspection de ceux-ci.

Immédiatement après sa constitution, une commission d'enquête industrielle doit examiner les questions qui lui sont déférées par le Ministre et s'efforcer d'exécuter son mandat; et s'il s'agit d'un conflit ou d'un différend dans lequel un règlement n'a pas été effectué entre temps, le rapport du résultat de ses enquêtes, y compris ses recommandations, doit être fait au Ministre dans les quatorze jours de sa nomination ou dans tel délai prorogé que celui-ci peut accorder à l'occasion.

Le ministre transmet des copies du rapport aux parties intéressées et publie le rapport de la manière qu'il juge appropriée.

\section{II-Loi concernant la conciliation et le travail}

La loi de la concilation et du travail, (chapitre 110, S.R. du Canada 1927), figure toujours dans les statuts, mais on ne voit plus très bien son utilité depuis l'adoption de la loi de 1948 sur les relations industrielles et sur Ies enquêtes visant les différends du travail.
En effet, cette dernière loi s'applique en particulier aux chemins de fer. Or la loi concernant la conciliation et le travail visait principalement les conflits qui pouvaient survenir dans le chemin de fer, tout en prévoyant des méthodes de règlement pour les autres conflits du travail, notamment l'intervention d'un amiable compositeur, la nomination d'arbitres, l'enregistrement de conseils de conciliation, etc.

Il ne fait pas de doute que les dispositions de la loi de 1948 en matière de règlement des conflits du travail jouissent en quelque sorte d'une priorité sur les dispositions de la loi concernant la conciliation et le travail. Les dispositions de la loi de 1948 exposées précédemment sont plus complètes et comportent des obligations plus précises que le chapitre 110.

Il est probable que le chapitre 110 a été conservé dans les statuts «par principe», étant donné que la loi concernant la conciliation et le travail constitue une des plus anciennes lois ouvrières du pays.

\section{B-LEGISLATION DE LA PROVINCE DE QUEBEC}

La législation de la Province de Québec en matière de conciliation et d'arbitrage s'inspire des mêmes principes que la législation fédérale. Les négociations collectives sont obligatoires entre les employeurs et les organisations de travailleurs certifiées par l'autorité compétente. Dans leurs grandes lignes, les mécanismes de conciliation et d'arbitrage fonctionnent d'une manière identique. Le droit de grève est retiré à certaines catégories d'employés des services publics, notamment ceux des corporations municipales et scolaires, mais par contre les sentences rendues par les tribunaux d'arbitrage sont exécutoires. 


\section{I-Loi des relations ouvrières 4}

\section{i) Intervention d'un conciliateur}

Lorsque les négociations entamées entre les parties se sont déroulées pendant trente jours sans succès ou si l'une ou l'autre des parties ne croit pas qu'elles puissent être complétées dans un délai raisonnable, chaque partie peut en donner avis à la Commission de relations ouvrières en exposant les difficultés rencontrées.

Sur réception d'un tel avis, la Commission en informe le ministre du travail qui charge un conciliateur de conférer avec les parties en vue d'aboutir à un règlement. Le conciliateur fait rapport dans les quatorze jours de la réception de ses instructions. Si le rapport indique que l'entente n'a pas été réalisée, le ministre nomme un conseil d'arbitrage suivant la Loi des différends ouvriers de Québec. Le rapport du conciliateur tient lieu de la demande prévue en ladite loi.

\section{ii) Interdiction de la grève et du lock-out}

Les grèves et les lock-outs dans tous les cas sont interdits avant quatorze jours après que le rapport d'un conseil d'arbitrage a été reçu par le Ministre du Travail. Ainsi que nous le verrons plus bas, les employés des services publics ne peuvent se mettre en grève. Voici le texte de l'article $24:$ : (1) Toute grève ou contregrève est interdite tant qu'une association de salariés n'a pas été reconnue comme représentant du groupe de salariés en cause et tant que cette association n'a pas fait les procédures voulues pour la conclusion d'une convention collective et qu'il ne s'est pas écoulé quatorze jours depuis la réception, par le ministre du travail,

(4) Loi instituant une commission des relations ouvrières, Chapitre $30(8 \mathrm{Geo}$. VI, 1944) modifiée par 9 Geo. VI, Chap. 44, 1945; 10 Geo. VI, Chap. 37, sanctionnée le 28 mars 1946. d'un rapport du conseil d'arbitrage sur le différend. Tant que les conditions ci-dessus n'ont pas été remplies, un employeur ne doit pas changer les conditions de travail de ses salariés sans leur consentement. (2) Toute grève ou contre-grève est interdite pendant la durée d'une convention collective tant que le grief n'a pas été soumis à l'arbitrage en la manière prévue dans ladite convention, ou à défaut de disposition à cette fin, en la manière prévue par la Loi des différends ouvriers de Québec (chap. 167), et que quatorze jours se sont écoulés depuis que la sentence a été rendue sans qu'elle ait été mise à effet. (3) Rien dans le présent article n'empêche une interruption de travail qui ne constitue pas une grève ou une contre-grève ». En d'autres termes, les conflits de droit comme les conflits d'intérêts doivent être soumis à une procédure obligatoire d'arbitrage avant que les parties puissent recourir à l'action directe.

\section{II-Loi des différends ouvriers de Québec 5}

La loi précise qu'aucun différend ou litige ne peut être soumis à un conseil de conciliation ou à un conseil d'arbitrage s'il y a moins de dix employés intéressés dans ce différend ou litige. Elle établit les fonctions du greffier des conseils de conciliation et d'arbitrage chargé du règlement des conflits du travail. Le greffier fait rapport au ministre du travail dans le plus bref délai possible. En fait, son intervention est maintenant déterminée et guidée par la Loi des relations ouvrières qui prévoit également l'intervention de conciliateurs.

\section{i) Conseils de conciliation}

Un différend peut être soumis à un conseil de conciliation dans les deux cas suivants, c'est-à-dire soit à la demande des patrons et des ouvriers,

(5) Loi pour régler les différends ouvriers, Chapitre 167, S.R.Q., 1941, modifiée par 12 Geo. VI, Chap. 27, 1948.

La Revue des relations industrielles 
soit à la demande d'une seule des parties.

Chaque conseil de conciliation est composé de quatre conciliateurs, chaque partie en nommant chacun deux.

Après avoir pris connaissance du différend et des faits, avoir entendu les parties et s'être efforcé de les concilier, le conseil de conciliation transmet son rapport au greffier. Après réception du rapport, le greffier, dans un délai qui ne doit pas excéder dix jours, transmet une copie certifiée du rapport aux parties intéressées. En l'absence d'un règlement, chaque partie peut requérir le greffier de porter le différend devant un Conseil d'arbitrage.

\section{ii) Conseils d'arbitrage}

Un conseil d'arbitrage est composé de trois membres, sujets Canadiens majeurs, nommés par le ministre. La partie employeur et la partie ouvrière doivent suggérer chacune une personne dans un délai de dix jours. A défaut, c'est le ministre du travail qui procède à la désignation. Les deux personnes ainsi désignées doivent $s^{\prime}$ entendre, dans un délai de cinq jours, pour désigner une troisième personne comme président du conseil d'arbitrage. Si elles ne peuvent s'entendre sur le choix de cette troisième personne, le ministre procède lui-même à la désignation.

Le conseil d'arbitrage peut être saisi d'une affaire qui a déjà été examinée par un conseil de conciliation ou d'un différend qui n'a pas été soumis à un tel conseil. Si la sentence d'un conseil d'arbitrage n'est pas acceptée et mise à exécution par les parties, celles-ci ou l'une d'elles ont le droit de soumettre le différend de nouveau à un conseil de conciliation.

Le conseil doit procéder à l'instruction du différend et rendre sa sentence avec toute la diligen $\sim$ possible, mais au plus tard dans les trois mois de la date de la nomination de son président. Si le conseil d'arbitrage le lui demande, en raison de la nature et des circonstances particulières du différend, le ministre du travail peut accorder, s'il le juge à propos, un délai supplémentaire qui ne doit pas excéder le délai suggéré par le conseil d'arbitrage.

La sentence est écrite et signée par la majorité des membres du conseil.

\section{iii) Acceptation de la sentence arbitrale}

Avant que la décision d'un conseil d'arbitrage soit rendue, les parties peuvent, en tout temps, convenir par écrit de s'en rapporter à la sentence du conseil d'arbitrage, de la même manière que les parties à un compromis fait sous l'empire du chapitre LXXIII du Code de procédure civile s'engageant de s'en rapporter à une sentence arbitrale. Tout engagement de cette nature donné par une partie doit être communiqué par le greffier à l'autre partie, et, si celle-ci consent également à s'en rapporter à la sentence, la décision du conseil d'arbitrage devient exécutoire.

\section{III-Loi concernant les différends entre les services publics et les salariés à leur emploi ${ }^{6}$}

Tout différend concernant les conditions de travail entre un service public et ses salariés doit être soumis à l'arbitrage suivant les dispositions de la convention collective existant entre ce service public et les représentants de ces salariés, s'il y en a une et si elle y pourvoit, et dans tout autre cas, suivant les dispositions de la Loi des différends ouvriers de Québec.

La sentence arbitrale, unanime ou majoritaire, peut être exécutée sous l'autorité d'un tribunal compétent sur poursuite intentée par une partie intéressée ou par la Commission de relations ouvrières laquelle n'est pas tenue de mettre en cause la partie

(6) Loi concernant les différends entre les services publics et leurs salariés, Chapitre 31, 8 Geo. VI, 1944. 
pour le bénéfice de laquelle elle agit.

Pour ce qui est des fonctionnaires visés par la loi du service civil, c'est la Commission du service civil qui joue envers eux le rôle d'un conseil d'arbitrage.

Toute grève ou lock-out est interdit en toute circonstance en ce qui concerne les emplovés des services publics.

Par services publics, on entend les corporations municipales et scolaires, les institutions d'assistance nublique, les asiles d'aliénés et les entreprises d'utilité publique et de transport, à l'exception des chemins de fer sous la juridiction du Parlement du Canada, les services du gouvernement de la province, mais à l'égard seulement des fonctionnaires et ouvriers visés par la Loi du service civil.

Des pénalités frappent les personnes qui violent les dispositions de la loi.

\section{IV-Loi concernant les corporations municipales et scolaires et leurs employés?}

Les conseils d'arbitrage qui sont saisis des différends entre les corporations municipales et scolaires et leurs employés sont composés de trois membres.

Tous les deux ans, au plus tard dans les trente jours précédant la fin de son année financière, toute corporation municipale doit recommander au ministre des affaires municipales une personne pour agir comme membre du conseil d'arbitrage chargé de prendre connaissance de tout différend entre elle et ses employés pendant les deux années financières suivantes. Les employés ou l'association accréditée pour les représenter doivent agir de la même manière.

Le conseil d'arbitrage entend et décide tous les différends qui peuvent s'élever, pendant la durée de son existence, entre la cornoration muni-

(7) Loi concernant les corporations municipales et scolaires et leurs employés, 13 Geo. VI, Chapitre 26, 1949. cipale pour laquelle il a été constitué et ses employés.

Les dispositions qui précèdent s'appliquent, mutatis mutandis, aux corporations scolaires. C'est évidemment le Secrétaire de la province qui agit lorsqu'il est question des corporations scolaires.

La loi des différends ouvriers de Québec stipule qu'en rendant sa sentence dans un différend intéressant une commission scolaire ou une corporation municipale, le conseil doit tenir compte de la situation financière de la corporation, de sa capacité de faire face aux obligations additionnelles qui peuvent résulter de la sentence et des impôts qui grèvent déjà les contribuables.

Nonobstant toute stipulation incompatible, aucune disposition d'une sentence arbitrale ou d'un jugement d'homologation comportant une augmentation de dépenses pour une corporation municipale ou scolaire n'est exécutoire avant l'expiration de son année financière en cours lors du prononcé de la sentence et ne peut rétrograder au delà de douze mois à compter de cette sentence.

La loi comporte également des précisions sur certaines clauses éventuelles de la sentence arbitrale ou de la convention collective, notamment en ce qui concerne la possibilité d'une clause de rajustèment automatique des salaires en fonction de l'indice du coût de la vie, l'obligation de ne pas venir en conflit avec les droits et pouvoirs attribués par la loi aux autorités municipales ou scolaires en matière d'engagement, de suspension et de renvoi de leurs employés.

Le Conseil d'arbitrage, stipule la Loi des différends ouvriers de Québec, ne peut fixer l'entrée en vigueur de la sentence avant le seizième jour qui suit la date de son émanation. S'il y a appel à la Commission municipale de Québec, l'exécution de la 
sentence est suspendue jusqu'à l'adjudication finale de la Commission municipale de Québec sur cet appel. L'appel doit être formé dans les quinze jours de la date de la sentence. La Commission entend les parties, complète la preuve si celle-ci est jugée insuffisante, et elle annule ou modifie la sentence si la chose s'avère nécessaire pour rencontrer la situation financière de la corporation municipale ou scolaire. Le décision de la Commission municipale de Québec est définitive.

\section{La Commission de relations ouvrières est-elle un tribunal judiciaire ?}

Me Mabie-Louis Beaulieu, c.R., avocat au Barreau de Québec

Un jugement très intéressant pour le monde du travail a été rendu par la Cour Supérieure à Québec, le 10 août dernier, dans une affaire qui mettait aux prises la Commission de relations ouvrières, une compagnie d'assurance comme employeur et un local de la Fédération américaine du travail comme syndicat de salariés. Il s'agit de l'arrêt prononcé par l'honorable juge Wilfrid Edge, sur une requête demandant l'émission d'un bref de Certiorari, pour suspendre l'xécution de trois décisions de la Commission de relations ouvrières à l'occasion d'une demande de reconnaissance syndicale: Cour Supérieure de Québec, no 58845, The Prudential Assurance Co. of America, requérante, vs juge L.-Conrad Pelletier, Alfred Charpentier, Elphège Beaudoin, H.-Conrad Lebrun et Pierre Audet, intimés et The National Federation of Insurance Agents' Council, American Federation of Labour, local 24538.

L'espèce était intéressante à plus d'un point de vue: quand un vote au scrutin secret doit-il être accordé par la Commission de relations ouvrières ? Quelle espèce de preuve la Commission doit-elle exiger pour appuyer ses décisions? Un membre de la Commission qui n'a pas pris part à l'audition de l'affaire peut-il parti- ciper à la décision ? Dans quels cas la Commission doit-elle reviser ou révoquer ses décisions, les ordres qu'elle a rendus ou les certificats qu'elle a émis? Quand y a-t-il abus ou excès de juridiction de la part de la Commission? La Commission estelle un tribunal judiciaire ou un organisme administratif ? Ce dernier point seulement a été décidé par la Cour. Vu lá décision à laquelle le tribunal en est venu sur ce point, il n'y avait pas lieu pour lui d'étudiè les autres questions, d'aller plus loin et d'examiner les griefs qu'on lui avait soumis contre les décisions de la Commission pour obtenir l'émission d'un bref de certiorari.

La requête était basée sur les articles 1292 et 1293 du Code de Procédure civile.

«1292. Dans tous les cas où l'appel n'est pas donné des tribunaux inférieurs mentionnés aux articles 59, 63,64 et 65 , le moyen d'évoquer la cause avant jugement ou de faire reviser le jugement rendu est le bref de certiorari à moins que ce recours même ne soit refusé par la loi. »

« 1293. Ce recours, néanmoins, n'a lieu que dans les cas suivants: 1 . Lorsqu'il y a défaut ou excès de juridiction; 2. Lorsque les règlements sur lesquels la plainte est portée ou le jugement rendu sont nuls ou sans 